\title{
Effect of ferric carboxymaltose on serum phosphate and C-terminal FGF23 levels in non-dialysis chronic kidney disease patients: post-hoc analysis of a prospective study
}

Merche Prats ${ }^{1,3^{*}}$, Ramon Font ${ }^{1}$, Carmen García ${ }^{1}$, Carmen Cabré ${ }^{1}$, Manel Jariod ${ }^{2}$ and Alberto Martinez Vea ${ }^{1}$

\begin{abstract}
Background: Some parenteral iron therapies have been found to be associated with hypophosphatemia. The mechanism of the decrease in serum phosphate is unknown. The aim of this study is to examine the effect of IV ferric carboxymaltose(FCM) on phosphate metabolism and FGF23 levels in patients with chronic kidney disease(CKD).

Methods: This is a post-hoc analysis of a prospective study carried out in 47 non-dialysis CKD patients with iron-deficiency anaemia who received a single $1000 \mathrm{mg}$ injection of FCM. Markers of mineral metabolism (calcium, phosphate, 1,25-dihydroxyvitamin D, PTH and FGF23[c-terminal]) were measured prior to FCM administration and at week 3 and week 12 after FCM administration. Based on the measured levels of serum phosphate at week 3, patiens were classified as hypophosphatemic or non-hypophosphatemic.

Results: Serum phosphate levels decreased significantly three weeks after FCM administration and remained at lower levels at week 12 (4.24 \pm 0.84 vs $3.69 \pm 1.10$ vs $3.83 \pm 0.68 \mathrm{mg} / \mathrm{dL}$, respectively, $\mathrm{p}<0.0001$. Serum calcium, PTH and 1,25-dihydroxyvitamin D did not change over the course of the study. Serum FGF23 decreased significantly from 442(44.9-4079.2) at baseline to 340(68.5-2603.3) at week 3 and 191.6(51.3-2465.9) RU/mL at week 12, $\mathrm{p}<0.0001$. Twelve patients were non-hypophosphatemic and 35 hypophosphatemic. FGF23 levels decreased in both groups, whereas no changes were documented in any of the other mineral parameters.
\end{abstract}

Conclusions: In non-dialysis CKD patients, FCM induces reduction in serum phosphate levels that persists for three months. FCM causes a significant decrease in FGF23 levels without changes to other bone metabolism parameters.

Keywords: Chronic kidney disease, Ferric carboxymaltose, Fibroblast growth factor 23, Hypophosphatemia, Iron deficiency anaemia

\section{Background}

Iron deficiency is common in non-dialysis chronic kidney disease (CKD) patients and is most pronounced in haemodialysis patients [1,2]. Supplementation with oral or intravenous (IV) iron is a common practice in this population. Oral iron therapy is limited by poor gastrointestinal absorption, frequent adverse events and low adherence to treatment. Therefore, IV iron is the preferred method of

\footnotetext{
* Correspondence: pfk21@hotmail.com

'Nephrology Service.Hospital Universitari de Tarragona Joan XXIII, Universitat Rovira i Virgili, Tarragona, Spain

${ }^{3}$ Servicio de Nefrología, Hospital Universitari de Tarragona Joan XXIII, Carrer del Doctor Mallafré Guasch 4, 43007, Tarragona, Spain

Full list of author information is available at the end of the article
}

iron replacement in these patients [3]. Nevertheless, high iron doses can lead to serious adverse consequences such as exacerbation of oxidative stress, inflammation, endothelial dysfunction, immune deficiency and increased tissue iron stores $[4,5]$. There are also other concerns about currently available IV iron agents, including the potential for immunogenic reaction, dose limitations and the requirement of a test dose, and the desired rate of repletion [6-8]. To overcome these limitations, new IV iron preparations have been introduced, offering higher single-dose options with an acceptable side-effect profile [7].

Ferric carboxymaltose (FCM) is an innovative nondextran iron complex that is composed of a ferric hydroxide 
core stabilized by a carbohydrate shell, carboxymaltose, allowing controlled delivery of iron to the target tissues [9]. Unlike earlier forms of IV iron, FCM can be given in a dose providing up to $1000 \mathrm{mg}$ of iron administered as a rapid infusion over $15 \mathrm{~min}$. without the need for a test dose [9]. In predialysis CKD patients and in patients undergoing haemodialysis, FCM is effective and well tolerated, and is associated with few adverse events $[8,10,11]$. A common adverse event associated with FCM is a transient, asymptomatic hypophosphatemia, which has been primarily reported in patients with postpartum iron-deficiency anaemia and in patients with iron-deficiency anaemia due to heavy uterine bleeding who were treated with large doses of FCM [12,13]. However, hypophosphatemia is neither widely acknowledged nor documented in CKD patients. In fact, transient hypophosphatemia has only been reported in $2.7 \%$ of non-dialysis CKD patients [9] and in 4.3\% of a CKD population [14] treated with FCM, but was not mentioned in the study conducted by Covic A et al. [8] on anaemic haemodialysis patients or in the study by Grimmelt $\mathrm{A}$ et al. [10] on predialysis CKD patients treated with variable doses of FCM.

The cause of hypophosphatemia during IV iron therapy remains unclear. It has been observed after the administration of other IV iron preparations such as iron saccharide [15] or iron polymaltose [16], but not with low molecular weight iron dextran [7], ferric gluconate [17] or iron isomaltoside [18]. Because hypophosphatemia has been reported in association with stimulated erythropoiesis in other haematopoietic disorders [19-21], it has been suggested that iron-induced hypophosphatemia might be the result of an increased cellular uptake of phosphate during erythropoiesis [13]. However, the main mechanism of ironinduced hypophosphatemia seems to be renal phosphate wasting [22]. Impaired tubular phosphate reabsorption has been reported during treatment with saccharated ferric oxide [15] and with iron polymaltose [16]. In addition to renal phosphate loss, an inhibition of renal $25(\mathrm{OH}) \mathrm{D} 1 \alpha$-hydroxylase activity and decreased 1,25-dihydroxyvitamin D levels were also reported in these cases $[15,16]$.

Since parenteral iron may have a direct toxic effect on proximal renal tubular cells, renal phosphate loss could be the consequence of proximal tubular dysfunction induced by iron therapy [23]. Nevertheless, the dual inhibition of tubular phosphate reabsorption and $1 \alpha$-hydroxylation of vitamin $\mathrm{D}$ observed during iron therapy, suggests that a phosphatonin hormone, fibroblast growth factor 23 (FGF23), may play a role in the hypophosphatemia induced by IV iron. In fact, hypophosphatemia with impaired tubular reabsorption, low $1,25(\mathrm{OH})_{2}$ vitamin D and high FGF23 levels has been reported in anaemic patients with normal renal function treated with saccharated ferric oxide or iron polymaltose $[15,16]$, supporting an etiologic role of FGF23 in the development of hypophosphatemia. In haemodialysis patients, IV low molecular weight iron dextran [24] or saccharate ferric oxide [25] also induce an increase in FGF23 levels and a decrease in PTH levels but no changes in serum phosphate.

To investigate the possible link between FCM therapy and phosphate metabolism, and to ascertain the possible mechanisms of FCM-associated hypophosphatemia, a post-hoc analysis was conducted on existing data from our previous study evaluating FCM therapy in a group of predialysis CKD patients with iron-deficiency anaemia [26].

\section{Methods}

\section{Patients}

This study was performed as a post-hoc analysis of a prospective study carried out in non-dialysis CKD patients treated with FCM [26]. Subjects $\geq 18$ years of age with an estimated glomerular filtration rate $<60 \mathrm{ml} / \mathrm{min} / 1.73 \mathrm{~m}^{2}$, serum haemoglobin $<11 \mathrm{~g} / \mathrm{dL}$ and either serum ferritin $<$ $100 \mathrm{ng} / \mathrm{dL}$ or transferrin saturation $<20 \%$, were included in the study. Exclusion criteria included oral or IV iron treatment or blood transfusion during the three-month period prior to the study, active infection, active bleeding, anticipated dialysis in the next three months, and known hypersensitivity to other IV iron preparations.

The study was approved by the ethics committee of the Joan XXIII University Hospital of Tarragona, and all participants provided written informed consent.

\section{Study design}

All patients received a single IV infusion of FCM (Ferinject $^{\mathrm{R}}$, Vifor Pharma, Switzerlan) over $30 \mathrm{~min}$. at a maximum dose of $1000 \mathrm{mg}(15 \mathrm{mg} / \mathrm{Kg})$ dilute in $250 \mathrm{~mL}$ normal saline, and were monitored for 12 weeks. During the study period, dietary phosphate intake and doses of erythropoiesis stimulating agents, active vitamin D, phosphate binders and cinacalcet hydrochloride were kept unchanged.

According to levels of serum phosphate obtained at week three, patients were classified as either hypophosphatemic or non-hypophosphatemic if phosphate levels decreased or remained unchanged or even increased compared to baseline levels.

\section{Biochemical determinations}

Blood samples were taken at baseline and at the end of week 3 and week 12 of the follow-up period. Serum haemoglobin, transferrin saturation, ferritin, calcium, phosphate and creatinine were determined by means of standard laboratory methods. Serum parathyroid hormone was determined by means of chemiluminescence(DPC Laboratories, Miami, FL, USA). 
Baseline levels of 25-hydroxy vitamin $\mathrm{D}$ were measured by chemiluminiscence(Diasorin, Italy). Serum $1,25(\mathrm{OH})_{2}$ D levels were measured by RIA(Diasource Immuno Assays, Belgium). Serum FGF23 concentrations were measured with the human FGF23 (C-terminal) ELISA kit (Immutopic, Inc, San Clemente, CA, USA). This C-terminal assay use two antibodies directed against the carboxyl-terminal portion of the molecule and detects both total intact and C-terminal FGF23 [27]. The estimated glomerular filtration rate(GFR) was calculated using the abbreviated MDRD formula [28].

\section{Statistical analysis}

Statistical analysis was performed using the SPSS/PC, v.15 statistical package (IMC, Chicago, IL). Values are expressed as mean \pm SD, and in case of PTH and FGF23 levels as median(range). Because serum FGF23 levels were not normally distributed, log-transformed values were used in the analysis. Mean levels were compared between time points using the repeated measurements ANOVA. The univariate relationship between variables was assessed by means of Spearman correlations. A p value of $<0.05$ was considered statistically significant.

\section{Results}

\section{Study population}

A total of 50 patients were enrolled in the study. Two subjects were withdrawn during the study because of bleeding, and one patient was excluded due to nonadherence to study protocol. Thus, 47 patients were included in the final analysis. The baseline demographics and clinical characteristics are shown in Table 1. Mean age was 72 years, and the most common primary renal disease was vascular nephropathy. Mean serum creatinine and estimated GFR were $2.5(0.9) \mathrm{mg} / \mathrm{dL}$ and $26.1(0.4) \mathrm{ml} / \mathrm{min} / 1.73 \mathrm{~m}^{2}$, respectively. Most patients (85\%) were in CKD stages 3-4. Only four patients were treated with phosphate binders, whereas 18 patients were receiving active vitamin D: paricalcitol(16 patients), calcitriol(2 patients). The mean cumulative dose of FCM was $971.7(69.3) \mathrm{mg}$. and there were no adverse events associated with FCM infusion during the study period.

\section{Effect of FCM on iron-deficiency anaemia}

Changes in iron indices and haemoglobin concentration before and after FCM are presented in Table 2. Haemoglobin levels and serum ferritin and transferrin saturation rose significantly and remained elevated at the end of the study.

\section{Effect of FCM on mineral parameters}

The biochemical parameters of mineral metabolism at baseline and after FCM infusion are shown in Table 2. Serum phosphate levels decreased significantly three 3 weeks after the FCM administration, and remained at lower levels at
Table 1 Baseline demographic and clinical characteristics

\begin{tabular}{ll}
\hline Characteristics & Value \\
\hline Age(years) & $72.2(11.6)$ \\
Male/Female & $23 / 25$ \\
Primary renal disease & \\
Vascular nephropathy & $23(49 \%)$ \\
Glomerulonephritis & $2(4 \%)$ \\
Interstitial nephropathy/polycystic kidney disease & $3(6 \%)$ \\
Diabetes & $12(26 \%)$ \\
Unknown & $7(15 \%)$ \\
Chronic kidney disease stage & \\
Stage 3 & $13(28 \%$ \\
Stage 4 & $27(57 \%)$ \\
Stage 5 & $7(15 \%)$ \\
Use of erythropoiesis stimulating agents & $23(49 \%)$ \\
Use of phosphate binders & $4(8.5 \%)$ \\
Use of active vitamin D & $18(38 \%)$ \\
Use of cinacalcet hydrochloride & $1(2 \%)$ \\
Serum creatine, mg/dL & $2.56(0.95)$ \\
eGFR, ml/min per 1.73 m² & $26.1(10.4)$ \\
Haemoglobin, g/dL & $10(0.7)$ \\
Ferritin, ng/mL & $67.8(61.7)$ \\
Transferrin saturation, \% & $14.6(6.4)$ \\
Calcium, mg/dL & $9.3(0.4)$ \\
Phosphate, mg/dL & $4.2(0.8)$ \\
PTH, pg/mL & $138.1(2.5-600)$ \\
25-hydroxyvitamin D, ng/ml & $12.8(6.6)$ \\
Fibroblast growth factor 23, RU/mL & $9.7(4.4)$ \\
\hline
\end{tabular}

week 12. Serum calcium, PTH and 1,25-vit D levels did not change during the study. However, serum FGF23 decreased significantly from $442(44.9-4079.2) \mathrm{RU} / \mathrm{mL}$ at baseline to 191.6(51.3-2465.9) $\mathrm{RU} / \mathrm{mL}$ at the end of the study. Changes in serum phosphate and C-terminal FGF23 were similar in all stages of CKD. There were no significant changes in estimated GFR during the study.

The relationship between serum phosphate levels and mineral metabolis parameters are shown in Table 3. A significant association was found between serum phosphate and FGF23 levels at week three. Serum phosphate was negatively correlated with estimated GFR throughout the study. There was no correlation between the decline in phosphate and FGF23 levels at week 3 or at week 12 .

Baseline serum FGF23 levels significantly correlated with phosphate $(\mathrm{r}=0.28, \mathrm{p}=0.05)$, transferrin saturation $(\mathrm{r}=-0.40, \mathrm{p}=0.001)$, ferritin $(\mathrm{r}=-0.30, \mathrm{p}=0.007)$ and 
Table 2 Effect of FCM on haemoglobin concentration, iron indices and mineral metabolism parameters

\begin{tabular}{llllc}
\hline & Baseline & Week 3 & Week 12 & p-value \\
\hline Haemoglobin, g/dL & $10(0.7)$ & $10.8(0.8)$ & $11.4(1.3)$ & $<0.0001$ \\
TSAT, \% & $14.6(6.4)$ & $28.9(10)$ & $25.6(12.7)$ & $<0.0001$ \\
Ferritin, $\mathrm{ng} / \mathrm{mL}$ & $67.8(61.7)$ & $502.5(263.3)$ & $230(144.6)$ & $<0.0001$ \\
Calcium, mg/dl & $9.3(0.4)$ & $9.3(0.5)$ & $9.3(0.5)$ & $\mathrm{ns}$ \\
Phosphate, mg/dL & $4.2(0.8)$ & $3.6(1.1)$ & $3.8(0.6)$ & $\mathrm{ns}$ \\
PTH, pg/ml & $138.1(2.5-600)$ & $124(2.5-736)$ & $106.5(2.5-613)$ & $\mathrm{ns}$ \\
$1,25(\mathrm{OH})_{2}$ D, pg/mL & $9.7(4.4)$ & $10(3.7)$ & $10.4(5.4)$ & $<0.0001$ \\
FGF23, RU/mL & $442(44.9-4079.2)$ & $340(68.5-2603.3)$ & $191.6(51.3-2465.9)$ & $<0.0001$ \\
\hline
\end{tabular}

TAST: transferrin saturation.

estimated $\operatorname{GFR}(\mathrm{r}=-0.48, \mathrm{p}=0.002)$. No correlation between FGF23 and calcium, 1,25-vit D or PTH was found.

Hypophosphatemic vs non-hypophosphatemic patients Twelve patiens were non-hypophosphatemic and 35 hypophosphatemic. Age, use of active vitamin D or phosphate binders, dose of infused FCM, baseline estimated GFR, haemoglobin concentration, iron index, and serum calcium, phosphate, PTH, 25-hydroxy vitamin D and 1.25 vit D levels were similar in both groups (Table 4). Nevertheless, there was a trend toward higher baseline FGF23 levels in non-hypophosphatemic than in

Table 3 Relationship between phosphate levels and other mineral metabolism parameters and estimated GFR

\begin{tabular}{|c|c|c|c|c|c|c|}
\hline & \multirow{2}{*}{\multicolumn{2}{|c|}{$\begin{array}{l}\text { Baseline } \\
\text { Phosphate }\end{array}$}} & \multirow{2}{*}{\multicolumn{2}{|c|}{$\begin{array}{l}\text { Week } 3 \\
\text { Phosphate }\end{array}$}} & \multirow{2}{*}{\multicolumn{2}{|c|}{$\frac{\text { Week } 12}{\text { Phosphate }}$}} \\
\hline & & & & & & \\
\hline & $r$ & p-value & $r$ & p-value & $r$ & p-value \\
\hline \multicolumn{7}{|l|}{ Baseline } \\
\hline Calcium & 0.04 & ns & & & & \\
\hline PTH & 0.16 & ns & & & & \\
\hline FGF23 & 0.28 & 0.05 & & & & \\
\hline $1,25(\mathrm{OH})_{2} \mathrm{D}$ & -0.29 & 0.05 & & & & \\
\hline eGFR & -0.62 & $<0.0001$ & & & & \\
\hline \multicolumn{7}{|l|}{ Week 3} \\
\hline Calcium & & & 0.01 & ns & & \\
\hline PTH & & & 0.01 & ns & & \\
\hline FGF23 & & & 0.47 & 0.001 & & \\
\hline $1,25(\mathrm{OH})_{2} \mathrm{D}$ & & & 0.20 & ns & & \\
\hline eGFR & & & -0.53 & $<0.0001$ & & \\
\hline \multicolumn{7}{|l|}{ Week 12} \\
\hline Calcium & & & & & -0.02 & ns \\
\hline PTH & & & & & -0.21 & ns \\
\hline FGF23 & & & & & 0.19 & ns \\
\hline $1,25(\mathrm{OH})_{2} \mathrm{D}$ & & & & & -0.16 & ns \\
\hline eGFR & & & & & -0.39 & 0.007 \\
\hline
\end{tabular}

hypophosphatemic patients, but it did not reach statistical significance. No correlation was demonstrated between the percentage reduction of serum FGF23 and that of serum phosphate at any time in either of these groups.

\section{Effect of vitamin D therapy on mineral metabolism parameters during FCM treatment}

Eighteen patients were receiving vitamin D therapy at the time of the study and 29 were not. Baseline mineral metabolism parameters and changes in calcium, phosphate, PTH and 1,25- vit D throughout the study did not differ between untreated and treated patients. However, the observed decrease in FGF23 levels in the group of patients treated with vitamin $\mathrm{D}$ was lower than that observed in the untreated patients: $-6.8 \%(-84.7 \%$ to $325.6 \%)$ and $-36.1 \%$ $(-91.6 \%$ to $52.4 \%)$ at week 3 , respectively, and $-21.7 \%$ $(-66.5 \%$ to $46 \%)$ and $-55.7 \%(-92.3 \%$ to $90.3 \%)$ at week 12 , respectively, $\mathrm{p}=0.03$.

\section{Discussion}

In this post-hoc analysis we showed that a single infusion of FCM in non-dialysis CKD patients causes significant and prolonged reduction in serum phosphate levels and a decrease in C-terminal FGF23 levels, but no changes in serum calcium, PTH or 1,25-vit D levels.

\section{FCM and hypophosphatemia}

Transient mild-moderate asymptomatic hypophosphatemia is common among patients receiving FCM, especially in patients with postpartum iron deficiency anaemia or uterine bleeding [12,13]. Hypophosphatemia has also been documented with other available IV iron preparations such as iron saccharide complexes [15] or iron polymaltose [16], but not with low molecular weight iron dextran [7] or iron isomaltoside [18]. The mechanisms of the decrease in serum phosphate are unknown, and the differences in the hypophosphatemic effect observed with various IV iron preparations remain unclear.

Several mechanisms have been suggested to explain the hypophosphatemia induced by IV iron therapy, including 
Table 4 Baseline characteristics of hypophosphatemic and non-hypophosphatemic patients

\begin{tabular}{|c|c|c|c|}
\hline & $\begin{array}{l}\text { Hypophosphatemic } \\
(\mathrm{n}=35)\end{array}$ & $\begin{array}{l}\text { Non-hypophosphatemic } \\
(n=12)\end{array}$ & $\mathrm{p}$-value \\
\hline Age(years) & $72.3(11.2)$ & $72.5(13.5)$ & ns \\
\hline Serum creatinine, mg/dL & $2.5(1)$ & $2.7(0.7)$ & ns \\
\hline eGFR, $\mathrm{ml} / \mathrm{min} / 1.73 \mathrm{~m}^{2}$ & $26.6(10.4)$ & $24.6(10.4)$ & ns \\
\hline Haemoglobin, g/dL & 10(0.6) & 10(0.8) & ns \\
\hline Ferritin, ng/mL & $64.1(58.3)$ & $78.5(72.2)$ & ns \\
\hline Transferrin saturation, \% & 14.6(6.4) & 14.6(6.7) & ns \\
\hline FCM, dosage $(\mathrm{mg})$ & $966.4(77.9)$ & 987(31.6) & ns \\
\hline Use of phosphate binders, $n(\%)$ & $3(8.5)$ & $1(8.3)$ & ns \\
\hline Use of active vitamin $\mathrm{D}, \mathrm{n}(\%)$ & $14(40)$ & $4(33.3)$ & ns \\
\hline Calcium, mg/dL & $9.3(0.4)$ & $9.3(0.6)$ & ns \\
\hline Phosphate, mg/dL & $4.1(0.8)$ & $4.5(0.7)$ & ns \\
\hline PTH, pg/mL & $147.8(31.6-600)$ & $84(2.5-404.9)$ & ns \\
\hline 25-hydroxyvitamin $\mathrm{D}, \mathrm{ng} / \mathrm{mL}$ & $12.5(6)$ & 13.8(8.5) & ns \\
\hline 1,25 dihydroxyvitamin $\mathrm{D}, \mathrm{pg} / \mathrm{mL}$ & $10.3(4)$ & $8.55(2)$ & ns \\
\hline Fibroblast growth factor $23, \mathrm{RU} / \mathrm{mL}$ & $437.5(44.9-2415)$ & 844.1(148.4-4079.2) & ns \\
\hline
\end{tabular}

the restoration of normal erythropoietic activity with cellular uptake of extracellular phosphate [13], phosphate binding to FCM via electrostatic interaction [29], and renal phosphate wasting due to the direct toxic effect of iron on renal tubular cells [22,23]. Finally, it has been suggested that FGF23, by reducing renal phosphate reabsorption and inhibiting $1 \alpha$-hydroxylation of vitamin $\mathrm{D}$, may play an etiologic role in the development of ironrelated hypophosphatemia $[15,16]$. In our study, the persistence of hypophosphatemia over 12 weeks despite the discontinuation of FCM argues against the extracellular uptake of phosphate or phosphate binding to FCM, as mechanisms of hypophosphatemia. We did not measure the fractional tubular reabsorption of phosphate, which may have allowed us to confirm the effect of FCM on renal phosphate transport.

It has been proposed that IV iron leads to hypophosphatemia by direct proximal tubular damage, resulting in a specific decrease in tubular phosphate reabsorption, but without other manifestations of a generalized proximal tubular toxicity, such as glucosuria or aminoaciduria [16]. The discontinuation of iron usually results in the rapid normalization of this disorder [25], suggesting that other mechanisms, rather than direct renal tubular damage, may be involved in the onset of hypophosphatemia. Furthemore, the high molecular mass of some IV iron preparations such as iron polymaltose (462,000 daltons) or FCM (150,000 daltons) may make their excretion through urine difficult, arguing against the direct toxic effect of iron on renal tubular cells [30]. However, it is possible that tubular damage induced by iron therapy is more likely in subjects with pre-existing CKD and anaemia treated with high doses of iron preparations. In fact iron accumulates in proximal tubular lysosomes in patients with proteinuria and/or chronic renal failure [31]. In rats subjected to a partial nephrectomy, the accumulation of iron in the cells of the proximal tubule was found to correlate with proteinuria, tubule damage and impairment of glomerular filtration [32]. Recently Tolouian et al. reported extensive deposits of iron in the mesangium and in the interstitial histiocytes in a patient with advanced diabetic nephropathy treated with ferumoxytol, another high molecular weight iron preparation, whereas renal iron deposition was not observed in another patient receiving IV iron dextran [33]. It seems that the intrinsic cytotoxic potential of IV iron may be dependend on the nature of the carbohydrate polymers employed, which appear to determine cellular iron uptake and the capacity of these compounds to induce cellular damage [34].

\section{FCM and FGF23}

In our study, FCM induces a decrease in FGF23 levels. This finding differs from previous studies which described an increased in intact FGF23 concentrations after IV iron therapy in patients with iron deficiency anaemia, normal renal function and normal baseline FGF23 levels $[15,16,30]$. In haemodialysis patients, IV saccharated ferric oxide or low molecular weight iron dextran resulted in an elevation in intact FGF23 levels accompanied by a reduction in PTH levels, but without changes in phosphate levels $[24,25]$. However, in a recent 
study in a dialysis population, a negative relationship was found between iron administration and serum intact FGF23 levels [35].

Our data suggest that in the CKD population, FCM has a negative effects on C-terminal FGF23 levels. Since FGF23 levels are highly regulated by serum phosphate or metabolic changes associated with hypophosphatemia [36], we can postulate that the resulting fall in phosphate levels as a consequence of iron-tubular toxicity would be expected to suppress FGF23 levels. However, in our study we did not find a relationship between the decline in phosphate and FGF23 levels, and changes in FGF23 levels did not differ between hypophosphatemic and non-hypophosphatemic patients, suggesting that the decrease in FGF23 was not related to the reduction in serum phosphate.

An unexpected finding in our study was the fact that FCM had no effect on 1,25-vit D or PTH levels. In subjects with normal renal function, FCM induces increased urinary fractional excretion of phosphate, decreased 1,25- vit D levels and increased PTH levels [37]. On the other hand, in hemodialysis patients, some iron preparations induce a reduction in PTH levels and no changes in 1,25- vit D levels [24,25]. It may be speculated that in CKD patients, the effect of intact FGF23 to suppress renal production of 1,25 vit D may be less pronounced, and the direct effect of FGF23 to suppress PTH secretion might be augmented.

Lower serum iron concentrations are associated with elevated FGF23 levels measured with a C-terminal FGF23 but not with an intact FGF23 assay [38,39]. It has been suggested that iron deficiency may result in an increased rate of proteolytic cleavage of the intact and biologically active hormone to inactive $\mathrm{C}$ - and $\mathrm{N}$ - terminal fragments $[40,41]$, although a recent western blotting analysis did not confirm this hypothesis [42].

In a population with iron deficiency, improvement in iron status with iron sulphate was associated with a decrease in plasma C-terminal FGF23 levels [43]. Thus, we suggest that FCM may have a negative effect on the production and/or secretion of FGF23 by bone cells. Iron overload is associated with osteoporosis, with a decrease in bone formation without changes in bone resorption [44]. Iron could slow down bone formation by inhibiting osteoblast progenitor cell differentiation [45]. FGF23 is expressed in osteoblast/osteocyte lineage cells [38], and osteoblastic bone formation is a potent modulator of FGF23 production and release into the bloodstream [46]. Therefore, we can postulate that if iron therapy has a negative effect on bone formation, it may also have a blunting effect on C-terminal FGF23 synthesis and secretion from osteoblasts. Finally, some iron preparations, such as FCM, may simultaneously inhibit FGF23 degradation in osteocytes, leading to an increase in intact FGF23 and reduced serum phosphate levels [37].

\section{FCM and vitamin D treatment}

In our study we also explored the influence of active vitamin D treatment on changes in FGF23 levels during FCM infusion. In the group of untreated vitamin D patients, FGF23 levels decreased, while this response was mitigated in the group of patients given vitamin D. These results are in keeping with the observation that vitamin D stimulates FGF23 production in osteoblasts [47], and also increases serum FGF23 levels in haemodialysis patients [48]. Therefore, we suggest that treatment with active vitamin $\mathrm{D}$ may play a role for maintaining elevated levels of FGF23 in non-dialysis CKD patients receiving FCM.

\section{Limitations of the study}

The strengths of this study include the use of a prospective sample of non-dialysis CKD patients treated with FCM with a long follow-up period, and uniform bone mineral metabolism data acquisition. There are, however, several potential limitations that deserve mention. Firstly, this was a secondary analysis of an open-label single-arm clinical trial that lacked a concurrent control group. These limitations prevented definitive conclusions to be drawn about the effect of FCM on serum phosphate in CKD patients. Secondly, we did not measure the fractional tubular reabsorption of phosphate and bone turnover markers were not available in our cohort. Thus, the direct effect of FCM on renal phosphate transport and bone metabolism and FGF23 synthesis could not be assessed. Thirdly, we did not determine the biologically active intact FGF23. This is important because of the known divergence of intact FGF23 and C-terminal FGF23 levels in the setting being studied, namely iron deficiency. Finally, the small sample size limited the ability of the study to analyse all of the factors that may have influenced the changes in phosphate levels.

\section{Conclusions}

Treatment with FCM for non-dialysis CKD patients with iron-deficiency anaemia induces reduction in serum phosphate levels that persists for three months. FCM causes a significant decrease in FGF23 levels without changes in other bone metabolism parameters. Further studies are needed to fully bring to light the mechanisms by which FCM modulates phosphate levels and disrupts FGF23 metabolism in CKD patients, and to determine whether long-term administration of FCM may induce hypophosphatemic osteomalacia in this population. 


\section{Competing interest}

The authors declared that they have no competing interest.

\section{Authors' contributions}

MP: Conception and design; Data analysis and interpretation; Provision of study patients; Manuscript writing; Final approval of manuscript. RF: Provision of study patients, Final approval of manuscript. CG: Provision of study patients; Final approval of manuscript, MJ: Data analysis and interpretation; Final approval of manuscript, AMV: Conception and design; Data analysis and interpretation; Provision of study patients; Manuscript writing; Final approval of manuscript. All authors read and approved the final manuscript.

\section{Acknowledgements}

The authors would like to thank Dr. Adriana Dusso for their comments and proofreading the manuscript.

\section{Author details}

${ }^{1}$ Nephrology Service.Hospital Universitari de Tarragona Joan XXIII, Universitat Rovira i Virgili, Tarragona, Spain. ${ }^{2}$ Unidad de Sistemas de Información de la Gestión, Hospital Universitari de Tarragona Joan XXIII, Tarragona, Spain. ${ }^{3}$ Servicio de Nefrología, Hospital Universitari de Tarragona Joan XXIII, Carrer del Doctor Mallafré Guasch 4, 43007, Tarragona, Spain.

Received: 14 March 2013 Accepted: 12 July 2013

Published: 31 July 2013

\section{References}

1. Hsu CY, McCulloch CE, Curhan GC: Iron status and haemoglobin level in chronic renal insufficiency. J Am Soc Nephrol 2002, 13:2783-2786.

2. Besarab A, Coyne DW: Iron supplementation to treat anemia in patients with chronic kidney disease. Nat Rev Nephrol 2010, 6:699-710.

3. Macdougall IC: Iron supplementation in the non-dialysis chronic kidney disease(ND-CKD) patients: oral or intravenous? Curr Med Res Opin 2010, 26:473-482.

4. Vaziri ND: Understanding iron: promoting its safe use in patients with chronic kidney failure treated by hemodialysis. Am J Kidney Dis 2013, 61:992-1000.

5. Rostoker G, Griuncelli M, Loridon C, Couprie R, Benmaadi A, Bounhiol C, Roy M, Machado G, Janklewicz P, Drahi G, Dahan H, Cohen Y: Hemodialysis-associated hemosiderosis in the era of erythropoiesis-stimulating agents: a MRI study. Am J Med 2012, 125:991-999.

6. Hörl WH: Clinical aspects of iron use in the anemia of kidney disease. J Am Soc Nephrol 2007, 18:382-393.

7. Auerbach $\mathrm{M}$, Ballard $\mathrm{H}$ : Clinical use of intravenous iron: administration, efficacy, and safety. Hematology Am Soc Hematol Educ Program 2010, 2010:338-347.

8. Covic A, Mircescu G: The safety and efficacy of intravenous ferric carboxymaltose in anaemic patients undergoing haemodialysis: a multi-centre, open-label, clinical study. Nephrol Dial Transplant 2010, 25:2722-2730

9. Lysen-Williamson KA, Keating GM: Ferric carboxymaltose: a review of its use in iron-deficiency anaemia. Drugs 2009, 69:739-756.

10. Grimmelt AC, Cohen CD, Fehr T, Serra AL, Wüthrich RP: Safety and tolerability of ferric carboxymaltose(FCM) for tratment of iron deficiency in patients with chronic kidney disease and in kidney transplant recipients. Clin Nephrol 2009, 71:125-129.

11. Qunibi WY, Martinez C, Smith M, Benjamin J, Mangione A, Roger SD: A randomized controlled trial comparing intravenous ferric carboxymaltose with oral iron for treatment of iron deficiency anaemia of non-dialysis-dependent chronic kidney disease patients. Nephrol Dial Transplant 2011, 26:1599-15607.

12. Seid MH, Derman RJ, Baker JB, Bnach W, Goldberg C, Rogers F: Ferric carboxymaltose injection in the treatment of postpartum iron deficiency anemia: a randomized controlled clinical trial. Am J Obstet Gynecol 2008, 199:435.e1-435.e7.

13. Van Wyck DB, Mangione AM, Morrison J, Hadley PE, Jehle JA, Goodnough $\mathrm{LT}$ : Large-dose intravenous ferric carboxymaltose injection for iron deficiency anemia in heavy uterine bleeding: a randomized, controlled trial. Transfusion 2009, 49:2719-2728.

14. Charytan C, Bernardo MV, Koch TA, Butcher A, Morris D, Bregman DB: Intravenous ferric carboxymaltose versus standard medical care in the treatment of iron deficiency anemia in patients with chronic kidney disease: a randomized, active-controlled, multi-center study. Nephrol Dial Transplant 2013, 28:953-964.

15. Shimizu Y, Tada Y, Yamaucho M, Okamoto T, Suzuki H, Ito N, Fukumoto S, Sugimoto T, Fujita T: Hypophosphatemia induced by intravenous administration of saccharated ferric oxide. Another form of FGF23-related hypophosphatemia. Bone 2009, 45:814-816.

16. Shouten BJ, Hunt PJ, Livesy JH, Frampton CM, Soule SG: FGF23 elevation and hypophosphatemia after intravenous iron polymaltose: a prospective study. J Clin Endocrinol Metab 2009, 94:2332-2337.

17. Imamura K: Effects of intravenous administration of iron preparations on the metabolism of phosphorus. Fukuoka Acta Med 1984, 75:316-326.

18. Kalra PA, Bock K, Meldal M: Iron isomaltoside 1000: a new high dose option for parenteral iron therapy. Port J Nephrol Hypert 2012, 26:13-24.

19. Mohammed S, Knoll S, Van Amberg A, Mennes PA: Cefotetan-induced haemolytic anemia causing severe hypophosphatemia. Am J Hematol 1994, 46:369-370.

20. Steiner M, Steiner B, Wilhelm S, Freund M, Schuff-Werner P: Severe hypophosphatemia during hematopoietic reconstitution after allogenic peripheral blood stem cell transplantation. Bone Marroww Transplant 2000, 25:1015-1016.

21. Sahara N, Tamashima S, Ihara M: Hereditary spherocytosis associated with severe hypophosphatemia in patients recovering from aplastic crisis. Rinsho Ketsueki 1998, 39:387-391.

22. Sanai T, Oochi N, Okada M, Imamura K, Okuda S, Ida M: Effect of saccharated ferric oxide and iron dextran on the metabolism of phosphorus in rats. J Lab Clin Med 2005, 146:25-29.

23. Sato K, Shiraki M: Saccharated ferric oxide-induced osteomalacia in Japan: iron-induced osteopathy due to nephropathy. Endocrine J 1998, 45:431-439.

24. Hryszko T, Rydzewska-Rosolowska A, Brzoski S, Koc-Zorawska E, Mysliwiec M: Low molecular weight iron dextran increases fibroblast growth factor-23 concentration together with parathyroid hormone decrease in hemodialyzed patients. Ther Apher Dial 2012, 16:146-151.

25. Takeda Y, Komaba H, Goto S, Fuji H, Umezu M, Hasegawa H, Fujimori A, Nishioka M, Nishi S, Fukagawa M: Effect of intravenous saccharated ferric oxide on serum FGF23 and mineral metabolism in hemodialysis patients. Am J Nephrol 2011, 33:421-426.

26. Prats M, Font R, Gutierrez, Nogués MR, Benito Y, Abejaro S, Garcia C, Romeu $M$, Martinez Vea A: Carboximaltosa férrica y estrés oxidativo en pacientes con enfermedad renal crónica prediálisis: efectos a corto y largo plazo. Nefrologia 2011, 31(Supp 2):58.

27. Imel EA, Peacock M, Pitukcheewanont P, Heller HJ, Ward LM, Shulman D, Kassem M, Rackoff P, Zimering M, Dalkin A, Drobny E, Colussi G, Shaker JL, Hoogendoorn EH, Hui SH, Econs MJ: Sensitivity of fibroblast growth factor 23 measurements in tumor-induced osteomalacia. J Clin Endocrinol Metab 2006, 91:2055-2061.

28. Levey AS, Coresh J, Greene T, Stevens LA, Zhang YL, Hendriksen S, Kusek JW, Van Lente F: Chronic Kidney Disease Collaboration: sing standardized serum creatinine values in the modification of diet in renal disease study equation for estimating glomerular filtration rate. Ann Intern Med 2006, 145:247-254.

29. Jahn MR, Andreasen $H B$, Fütterer $S$, Nawroth $T$, Schünemann $V$, Kolb U, Hofmeister W, Muñoz M, Bock K, Meldal M, Langguth P: A comparative study of the physicochemical properties of iron isomaltoside 1000 $\left(\right.$ Monofer ${ }^{R}$ ), a new intravenous iron preparation and its clinical implications. Eur J Pharm Biopharm 2011, 78:480-491.

30. Schoulten BJ, Doogue MP, Soule SG, Hunt PJ: Iron polymaltose-induced FGF23 elevation complicated by hypophosphatemic osteomalacia. Ann Clin Biochem 2009, 46:167-169.

31. Nankivell BJ, Boadle RA, Harris DCH: Iron accumulation in human chronic kidney disease. Am J Kidney Dis 1992, 20:580-584

32. Nankivell BJ, Tay Y-C, Boadley RA, Harris DCH: Dietary protein alters tubular iron accumulation after partial nephrectomy. Kidney Int 1994, 45:1006-1013.

33. Tolouian R, Rajabi B, Boman D, Bilbao J, Gupta J: Iron infusion and deposition in the kidney. Clin Nephrol 2013, 79:237-240.

34. Zager RA, Johnson AC, Hanson SY: Parenteral iron nephrotoxicity: Potential mechanisms and consequences. Kidney In 2004, 66:144-156.

35. Deger SM, Erten Y, Pasaoglu OT, Derici UB, Reis KA, Onec K, Pasaoglu H: The effects of iron on FGF23-mediated Ca-P metabolism in CKD patients. Clin Exp Nephrol 2013, 17:416-423.

36. Endo I, Fukumoto S, Ozono K, Namba N, Tanaka H, Inove D, Minagawa M, Sugimoto T, Yamauchi M, Michigami T, Matsumoto T: Clinical usefulness of 
measurement of fibroblast growth factor 23(FGF23) in hypophosphatemic patients: proposal of diagnostic criteria using FGF23 measurement. Bone 2008, 42:1235-1239.

37. Wolf M, Koch TA, Bregman DB: Effects of iron deficiency anemia and its treatment on fibroblast growth factor 23 and phosphate homeostasis in women. J Bone Miner Res 2013, 28:1793-1803.

38. Imel EA, Peacock M, Gray AK, Padgett LD, Hui SL, Econs MJ: Iron modifies plasma FGF23 differently in autosomal dominant hypophosphatemic rickets and healthy humans. J Clin Endocrinol Metab 2011, 96:3541-3549.

39. Farrow EG, Yu X, Summers $L$, Davis SI, Fleet JC, Allen MR, Robling AG, Stayrook KR, Jideonwo V, Magers MJ, Garringer HJ, Vidal R, Chan RJ, Goodwin CB, Hui SL, Peacock M, White KE: Iron deficiency drives an autosomal dominant hypophosphatemic rickets(ADHR) phenotype in fibroblast growth factor-23(Fgf23) knock-in mice. Proc Natl Acad Sci USA 2011, 108:E1146-E1155.

40. Durham BH, Joseph F, Bailey LM, Fraser WD: The association of circulating ferritin with serum concentrations of fibroblast growth factor-23 measured by three commerical assays. Ann Clin Biochem 2007, 44:463-466.

41. Bhattacharyya N, Wiench M, Dumitrescu C, Connolly BM, Bugge TH, Patel HV, Gafni RI, Cherman N, Cho M, Hager GL, Collins MT: Mechanism of FGF23 processing in fibrous dysplasia. J Bone Miner Res 2012, 27:1132-1141.

42. Braithwaite B, Bruggraber SF, Prentice A: Intact fibroblast growth factor-23 and fragments in plasma from gambian children. Osteoporos Int 2013, 24:1121-1124

43. Braithwaite B, Prentice AM, Doherty C, Prentice A: FGF23 is correlated with iron status but not with inflammation, and decrease after iron supplementation: a supplementary study. Int J Pediatr Endocrinol 2012, 1:27.

44. Yamasaki K, Hagiwara H: Excess iron inhibits osteoblast metabolism. Toxicol Lett 2009, 191:21-215.

45. Yang Q, Jian J, Abramson SB, Huang X: Inhibitory effects of iron on bone morphogenetic protein.2-induced osteoblastogenesis. J Bone Miner Res 2011, 26:1188-1196.

46. Samadfam R, Richard C, Nguyen-Yamamoto L, Bolivar I, Goltzman D: Bone formation regulates circulating concentrations of fibroblast growth factor 23. Endocrinology 2009, 150:4835-4845.

47. Liu S, Tang W, Zhou J, Stubbs JR, Luo Q, pi M, Quarles LD: Fibroblast growth factor 23 is a counterregulatory phosphaturic hormone for vitamin D. J Am Soc Nephrol 2006, 17:1305-1315.

48. Hansen D, Rasmussen K, Pedersen SM, Rasmussen LM, Brandi L: Changes in fibroblast growth factor 23 during treatment of secondary hyperparathyroidism with alfacalcidol or paricalcitol. Nephrol Dial Transplant 2012, 27:2263-2269.

doi:10.1186/1471-2369-14-167

Cite this article as: Prats et al:: Effect of ferric carboxymaltose on serum phosphate and C-terminal FGF23 levels in non-dialysis chronic kidney disease patients: post-hoc analysis of a prospective study. BMC Nephrology 2013 14:167.

\section{Submit your next manuscript to BioMed Central and take full advantage of:}

- Convenient online submission

- Thorough peer review

- No space constraints or color figure charges

- Immediate publication on acceptance

- Inclusion in PubMed, CAS, Scopus and Google Scholar

- Research which is freely available for redistribution

Submit your manuscript at www.biomedcentral.com/submit 